\title{
Desenvolvimento de uma Interface de Controle Assistiva Independente Baseada em Utilidade
}

\author{
Alexandre L. C. Bissoli ${ }^{1}$, Flavio Ferrara ${ }^{2}$, Teodiano F. Bastos-Filho ${ }^{1}$ \\ ${ }^{1}$ Laboratório de Automação Inteligente - Programa de Pós-Graduação em Engenharia \\ Elétrica - Universidade Federal do Espírito Santo (UFES) \\ Av. Fernando Ferrari, 514, Goiabeiras, 29075-910Vitória-ES \\ ${ }^{2}$ Politecnico de Milano - Piaaza Leonardo da Vinci \\ 20133, Milano, Italia \\ alexandre-bissoli@hotmail.com, femferrara@gmail.com, \\ teodiano.bastos@ufes.br
}

\begin{abstract}
An important, often underestimated problem while realizing an assistive control technology by means of biosignals is the proper design of the control interface. The principles and choices that guide this phase will sensibly affect the user interaction with the system. In this work, we show that a realistic performance-driven analysis can improve the interaction between user and interface, leading to a greater usefulness of the assistive system. Within the Brain-Computer Interface field, a metric called "BCI Utility" has been proposed in order to indicate the overall performance of the BCI system in a user-centered way.
\end{abstract}

Resumo.Um problema importante, muitas vezes subestimado durante a realização de uma tecnologia de controle assistivo por meio de sinais biológicos é o projeto adequado da interface de controle. Os princípios e as escolhas que norteiam esta fase afetam sensivelmente a interação do usuário com o sistema. Neste trabalho, mostra-se que uma análise orientada por desempenho realista pode melhorar a interação entre o usuário ea interface, proporcionando uma maior utilidade do sistema de apoio. Dentro do campo de Interface Cérebro-Computador, uma métrica chamada "BCI Utility"é proposta a fim de indicar o desempenho global do sistema de ICC de forma centrada no usuário.

\section{Introdução}

Durante as últimas décadas houve um grande avanço nas tecnologias que podem proporcionar melhorias na vida das pessoas com deficiência. Estas tecnologias, chamadas tecnologias assistivas, são equipamentos, produtos ou sistemas utilizados para aumentar, manter ou melhorar as capacidades funcionais das pessoas com deficiência [Cook e Polgar 2013].

O conceito de Interface Cérebro-Computador (ICC) surgiu com o objetivo de proporcionar a comunicação alternativa e controle para os indivíduos com deficiências motoras severas [Wolpawet al. 2002]. A arquitetura funcional de um sistema de ICC foi formalizada por Mason e $\operatorname{Birch}(2003)$. O modelo permite identificar os principais componentes envolvidos em um sistema de ICC genérica e como eles estão 
relacionados. Além disso, abre espaço tanto para ICC quanto para sistemas de controle não-ICC. O bloco funcional responsável pela tradução dos sinais biológicos do usuário, eletromiografia de superfície (sEMG) e de eletro-oculografia (EOG), em estados lógicos discretos será referido como tradutor de sinais biológicos (TSB).

A separação funcional entre o TSB e a Interface de Controle (IC) sugere que o primeiro deve fornecer saídas lógicas, independentes de qualquer conhecimento sobre como a IC traduzirá posteriormente aquelas saídas para o controlador do dispositivo. Segue que, para fins de comparação com outros TSBs, é necessário utilizar umaIC comum. A construçãodo sistema com uma forma semântica independente proporciona várias vantagens em aplicações assistivas:em primeiro lugar, a possibilidade de adaptar o paradigma TSB de acordo com o grau de incapacidade do indivíduo. Em segundo lugar, a ICbem concebida permite a comunidadecientífica comparar objetivamente as tecnologias TSB já existentes e facilitar o progresso do campo. No entanto, enquanto várias pesquisas são voltadas para a melhoria das técnicas de TSB, pouca atenção tem sido dada às metodologias e boas práticas para a concepção de tais interfaces de controle.

O problema de calcular um indicador capaz de expressar o desempenho global do sistema de ICC foi abordado por Seno, Matteucci e Mainardi [2010]. Os autores afirmaram que as métricas de desempenho habituais, tais como a precisão da classificação ou taxa de transferência de informações (do inglês informationtransfer rate, ITR) [Wolpawet al. 2002] são irrealistas e, mais importante, não levam em conta a concepção da IC e como ela interpreta a saída doTSB. Por estas razões,foi proposta uma métrica denominadaBCI Utility (em português, ICC Utilitária), que supera as limitações descritas anteriormente e permite comparar sistemas diferentes em uma perspectiva mais centrada no usuário.

Neste trabalho, BCI Utility é utilizada na concepção de uma simples IC baseada em menus. A seguir, será utilizado simplesmente o termo Utility para esclarecer que a métrica não se limita aos sistemas ICC. A componente IC fará parte de um sistema de apoio multimodal para dispositivos operando em um ambiente inteligente[Bissoli et al. 2015a] [Bissoli et al. 2015b].

O presente artigo está estruturado da seguinte forma: a Seção 2 apresenta o desenvolvimento da Interface de Controle, destacando a arquitetura funcional do sistema e as abordagens de interação; a Seção 3 introduz o conceito de Computação Utilitária e seus desdobramentos; a Seção 4 apresenta os resultados e compara a métrica proposta com a métrica tradicional; por fim, a Seção 5 aborda as considerações finais e apresenta algumas propostas de trabalhos futuros.

\section{Desenvolvimento da Interface de Controle}

O objetivo da utilização da interface consiste na integração de uma cadeira de rodas com um ambiente inteligente [Bissoli et al. 2015a]. O sistema permite a interação com vários dispositivos em um ambiente, por exemplo, uma sala, por meio de sinais biológicos, lidos a partir da cadeira de rodas, conforme esquematizado na Figura 1 [Bissoli et al. 2015b]. 


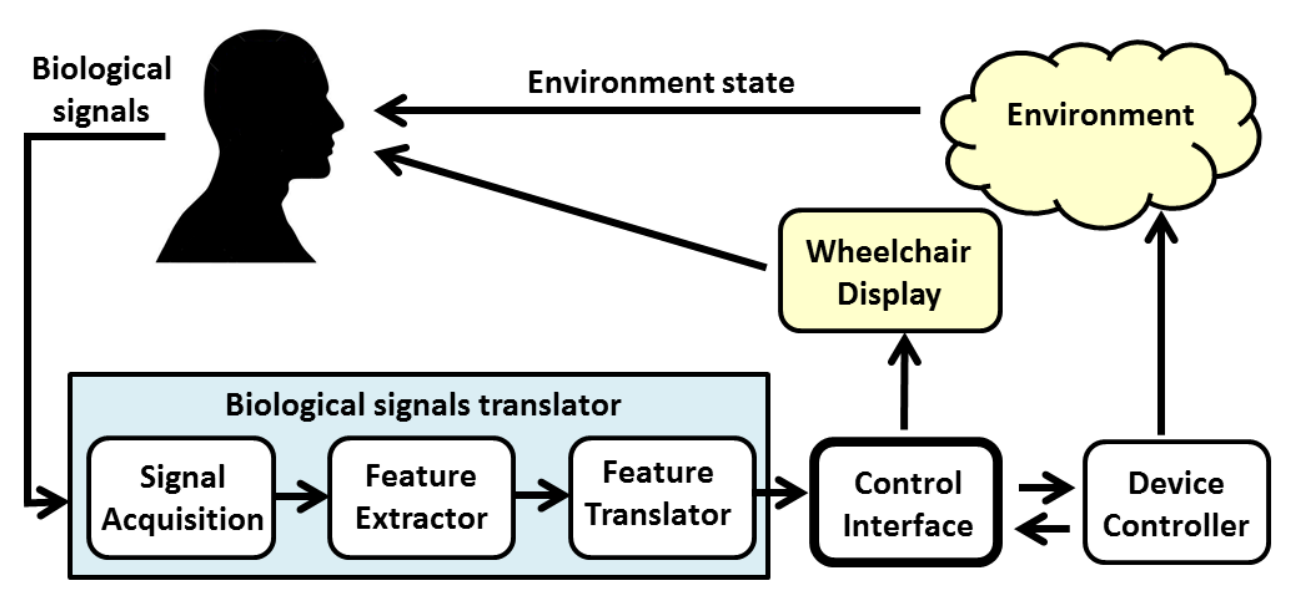

Figura 1.Arquitetura funcional do sistema assistivo.

Ao decodificar a intenção do usuário, o tradutor de sinais biológicos emite uma saída de tempo discreto que representa a classe reconhecida. A partir de agora, a saída do tradutor será referida apenas como comandos lógicos ou apenas comandos. O usuárioirá interagir com o sistema através de uma interface gráfica mostrada no monitor da cadeira de rodas (Figura 2).

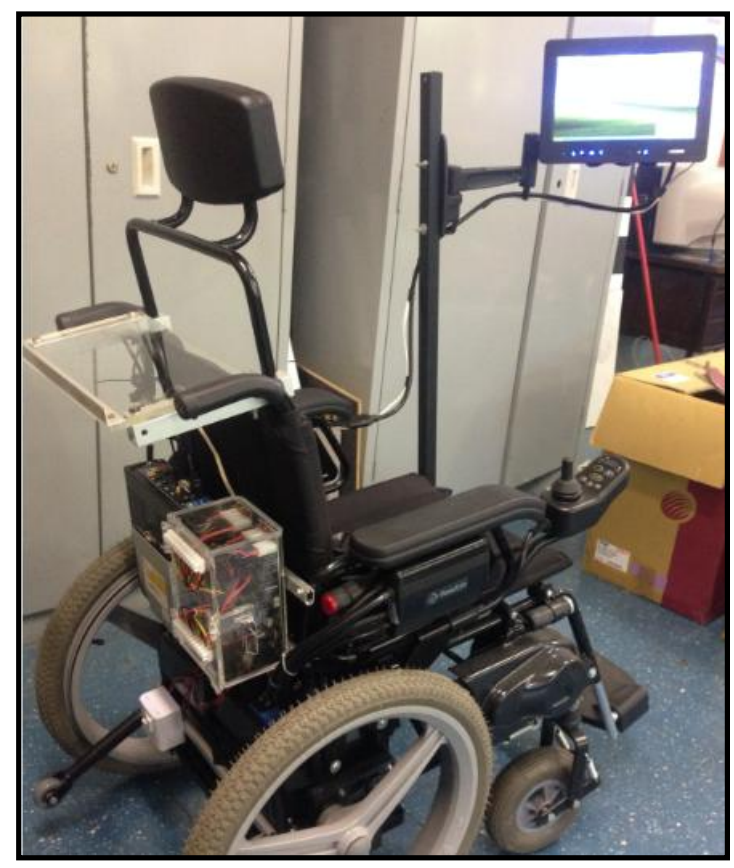

Figura 2.Cadeira de Rodas Motorizada do Laboratório de Automação Inteligente da UFES.

O controlador é executado em um Arduino e se comunica com o ambiente inteligente através de rádio frequência ou infravermelho[Bissoli et al. 2015a]; os detalhes de implementação estão fora do escopo deste artigo.

Como relatado em MasoneBirch(2003), uma característica importante para tornar o usuário mais independente é a de proporcionar um mecanismo para ligar e desligar o sistema. O caso de ligar é difícil e muito dependente do tipo de tradutor de 
sinais biológicos que o usuário está realmente usando. Muitas técnicas foram propostas; por exemplo, uma forma de ligar o sistema com o piscar dos olhos ao usar um controle baseado em eletroencefalografia (EEG) [Kirkupet al. 1997]. Então, essa tarefa serádeixada para o TSB e fora do projeto da IC. Em contrapartida, desligar o sistema será uma das opções que o usuário poderá escolher, enquanto o sistema está em execução. Ao mesmo tempo, é oferecida a possibilidade de o TSBdesligar de forma independente o sistema quando desejado.

\subsection{Interface de Controle}

O projeto da IC foi baseado em dois princípios fundamentais:

P1. O usuário deve ser capaz de realizar todas as tarefas possíveis, ou seja, operar qualquer dispositivo e desligar o sistema, com um conjunto muito limitado de comandos lógicos.

P2. O comportamento da interface desempenha um papel essencial na determinação do bom compromisso entre o desempenho eo número de comandos.

A fim de cumprir P1, optou-se por uma interface baseada em menu. A escolha dessa abordagem permite criar um mapeamento semântico entre os sinais de controle lógico do TSB em sinais de controle semântico que sejam significativos para a Interface de controle [MasoneBirch2003]. Um exemplo é a tradução do mesmo comando lógico Ligarem sinais de controle semânticos como Ligar a televisão e Ligar o rádio.

Este tipo de multiplexação é particularmente importante em tecnologias de apoio, nas quais o usuário possa ficar confuso ou cansado pela presença de vários comandos possíveis. Além disso, o método de classificação utilizado no recurso TSB provavelmente apresenta um desempenho inferior, com um número crescente de classes. Por outro lado, um menu tem algumas desvantagens de desempenho que devem ser levadas em consideração. Intuitivamente, o gargalo é representado pela opção mais distante da inicialmente apontada.

A Figura 3 apresenta um exemplo de como a IC é apresentada ao usuário no visor da cadeira de rodas, mostrando as opções disponíveis no ambiente inteligente. A opção selecionada é destacada no centro, enquanto as outras possibilidades estão listadas na parte inferior da interface. Apenas uma opção pode ser destacada, e atuada, em um determinado momento.

A Figura 4 apresenta um submenu da interface, que é aberto quando o usuário seleciona a opção Televisão.

O próximo passo é descobrir o número ideal de comandos lógicos. Essa escolha afeta a maneira em que o usuário irá navegar pelo menu. Em particular, a principal alternativa é entre navegação unidirecional e navegação bidirecional. 


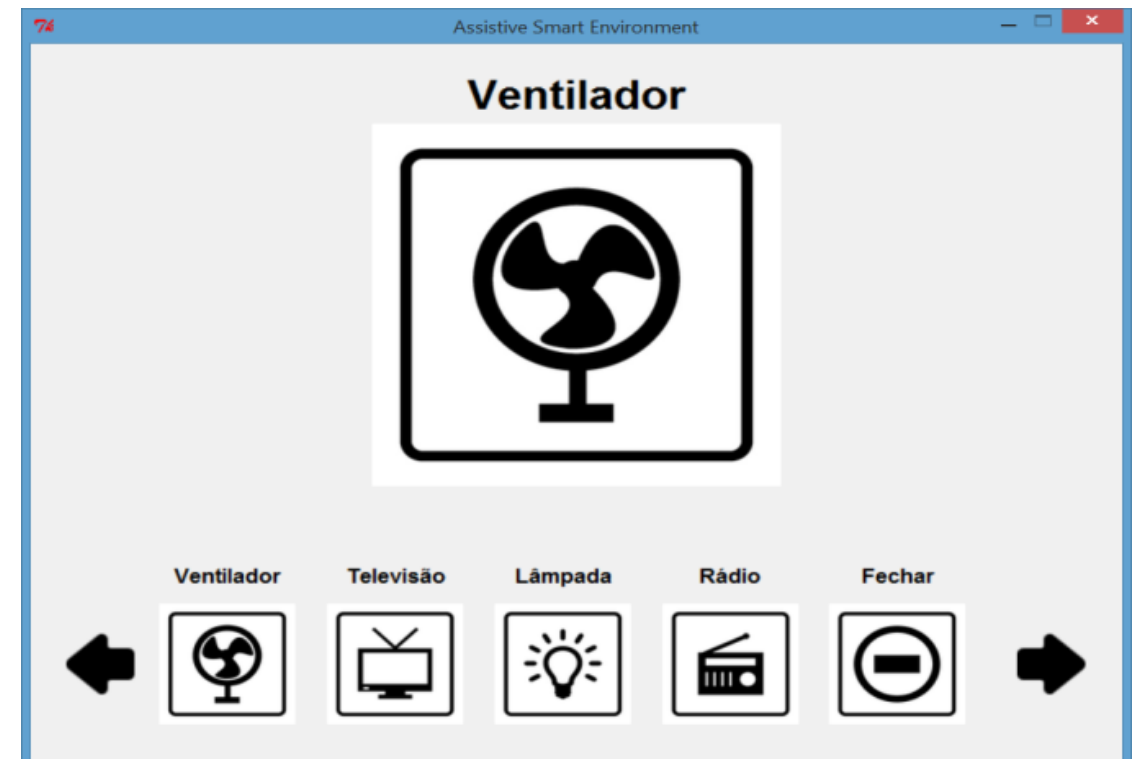

Figura 3. Menu da Interface de Controle.

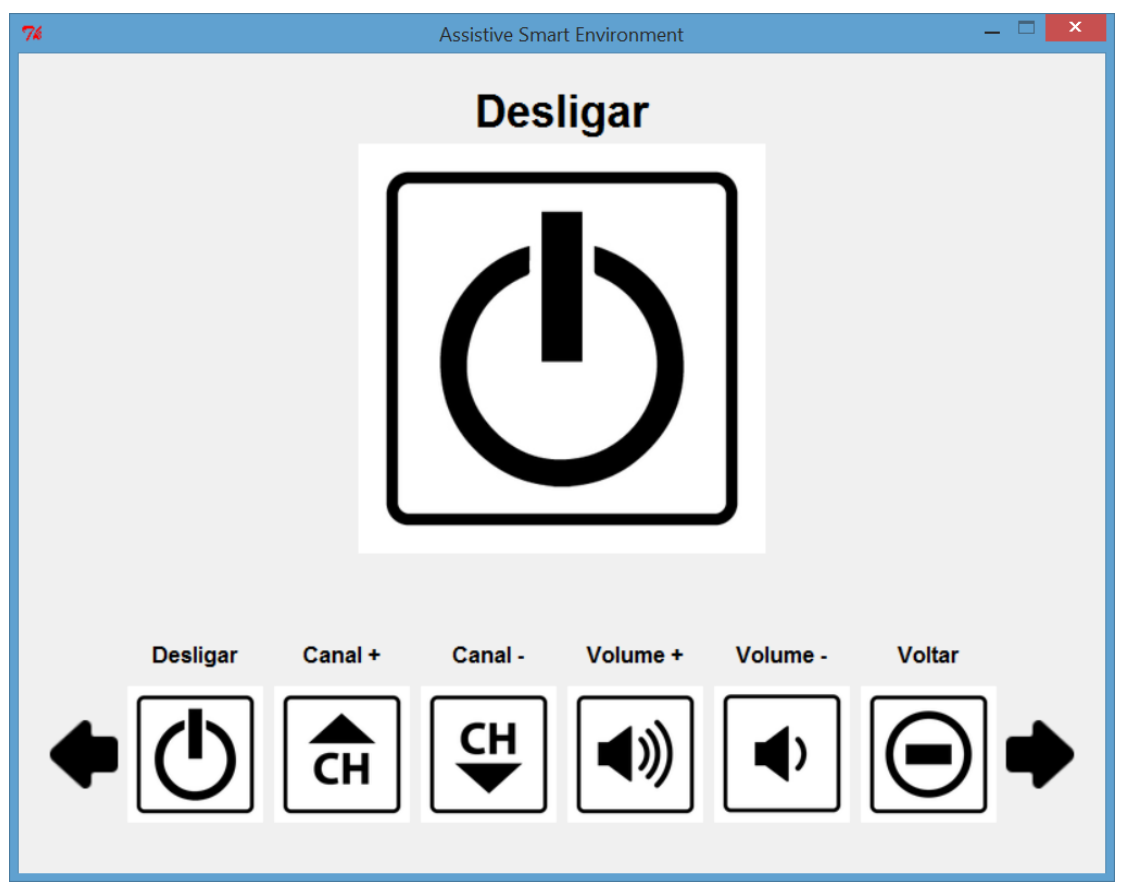

Figura 4. Submenu Televisão da Interface Gráfica.

\subsection{Abordagens de Interação}

Vamos considerar uma configuração com três comandos. Eles podem ser identificados comoDo (faça), Next (próximo) e Previous (anterior). O comportamento é claro: o comando Do opera o dispositivo representado pela opção destacada no momento, invertendo seu estado atual; o comando Next destaca a opção seguinte na sequência,e o comando Previous destaca a opção anterior. Se a opção destacada no momento é a opção mais à direita da interface(última), a próxima será aopção mais à esquerda da interface (primeira), e vice-versa. 
Foram comparados os resultados obtidos nessa configuração com a que considera outras abordagens de navegação. Uma abordagem mais simples que só permite a navegação unidirecional (ou seja, não incluindo o comando Previous), e uma terceira alternativa, onde o comando Previous é substituído por um comando Undo (desfazer). No segundo caso, o comportamento de desfazer é definido como o estado anterior à última ação executada.

\section{Computação Utilitária}

O conceito de Utilidade (Utility) pode ser expresso como o benefício médio $b_{d}$ trazido por qualquer dispositivo operado corretamente dividido pelo tempo esperado $T_{d}$ necessário para operá-lo.

Da mesma forma que Seno, Matteucci, eMainardo(2010), consideram-se as seguintes hipóteses para simplificar a derivação:

A1. A precisão $p$ do tradutorde sinais biológicos é constante ao longo dostestes.

A2. Cada teste é independente do anterior.

A3.Os dispositivos diferentes têm a mesma probabilidade de seremselecionados.

Suponha que a ICapresente $N$ opções. A fim de ser comparável com o clássico ITR, mede-se o benefício em bits. Baseado em A3, a informação transportada pelo dispositivo de escolha consiste em $b_{d}=\log _{2} N$ bits. Vale a pena notar que ITR não é afetado pelas características da IC. Assim, a informação ITR considera apenas o número de comandos lógicos do TSB, que, em geral, será diferente de $N$.

O tempo $T_{d}$ está relacionado com a distância $d$ entre a opção atual e a opção desejada. Para selecionar o dispositivo desejado, o usuário precisa navegar a distânciadna interface utilizando os comandos Next ou Previous, e em seguida selecionar o dispositivo desejado com o comando $D o$, levando a $d+1$ o total de testes.

Assumindo $c$ a duração de um único teste, com uma precisão de $p$, obtém-se o tempo esperado $T_{c}$ necessário para completar um comando:

$$
T_{c}=p c+(1-p)\left(c+T_{r}+T_{c}^{(1)}\right)
$$

onde $T_{r}$ é o tempo esperado para recuperar um erro. De acordo com a primeira abordagem, é fácil ver que apenas um comando adicional é necessário para desfazer uma classificação errada. Considerando A1 e A2, temos $T_{r}=T_{c}^{(l)}=T_{c}$

$$
T_{c}=c+(1-p) 2 T_{c} .
$$

Pode-se demonstrar que $T_{d}$ irá convergir para

$$
T_{d}=(d+1) T_{c}=\frac{(d+1) c}{2 p-1}
$$

se $p>0.5$. Se esta condição não for cumprida, o tempo esperado para operar corretamente um dispositivo vai a infinito e o sistema não é utilizável. 
A partir da fórmula geral de Utilidade e da Equação (3), definindo $b_{d}=\log _{2} N$, obtém-se finalmente:

$$
U=\frac{(2 p-1) \log _{2} N}{(d+1) c}
$$

Note que, utilizando os três comandos considerados, $d$ assumiria valores inteiros se $N$ é par, ou se $N$ é ímpar.

Com uma análise semelhante, calcula-se o tempo esperado eo valor Utility para as outras abordagens anteriormente introduzidas.

A avaliação de $T_{r}$ revela a principal desvantagem da segunda abordagem ou unidirecional (m): se um comando Do é mal interpretado como Next, o usuário terá de realizar pelo menos $N$ comandos para recuperar o erro.

Considerando os dois tipos de erro, com igual probabilidade, obtêm-se

$$
T_{c}^{(m)}=c+(1-p)\left(\frac{N+2}{2} T_{c}^{(m)}\right) .
$$

Quando se comparamas Equações (2) a (5), segue que, para $N>2$, o tempo de comando será maior na segunda abordagem (unidirecional) do que na primeira (bidirecional).

Na terceira abordagem, em queo comando Previous é substituído pelo Undo, o mesmo resultado é obtido para o exemplo analisado. No entanto, esta abordagem ainda é pior que a primeira considerada, pois $d$ terá valores $[0, N]$.

\section{Resultados}

Com esse trabalho foi possível simular um verdadeiro tradutor de sinais biológicos (TSB) com precisão $p$ e calcular os valores de utilidade (Utility) para as abordagens apresentadas. Considere, por exemplo, uma interface com $N=6$ opções; a duração do teste $c$ será assumida unitária, de modo que o valor calculado pode ser imediatamente comparado com ITR. Como ilustrado anteriormente, o método Utility é dependenteda opção desejada e da distânciad entre a opção desejada e a opção atual. A Figura 5 mostra essa dependência.

As três curvas representam as três abordagens de interação, respectivamente. Em todos os casos, a primeira opção corrente é destacada, de modo que a distância da mesma é igual a zero. Usando a navegação unidirecional com ou sem a possibilidade de desfazer o comando, $d$ varia de zero a cinco; em contrapartida, com a introdução do comando Previous, a sexta opção estará a uma distância digual a 1.

Pode-se observar uma redução no desempenho ao tentar operar dispositivos mais distantes, em termos da distância da opção. No entanto, ao permitir a navegação em ordem inversa, a abordagem bidirecional atenuaessa queda do desempenho e, no pior dos casos,ela acontece para $d=\frac{N}{2}$. Para mostrar o desempenho global, considera-se uma Utility média como a média aritmética da Utility para os possíveis valores de distância $d$. 
É importante notar que a recursividade implicada em (2) e (5) comporta um limiar inferior,abaixo do qual o sistema não pode ser usado. A Figura 6 mostra a diferença entre aUtility média para diferentes abordagens de navegação. Para $p<0,5$ a saída é mais provável errada do que correta, então o sistema iráoperar um dispositivo indesejado ou navegar na direção oposta.
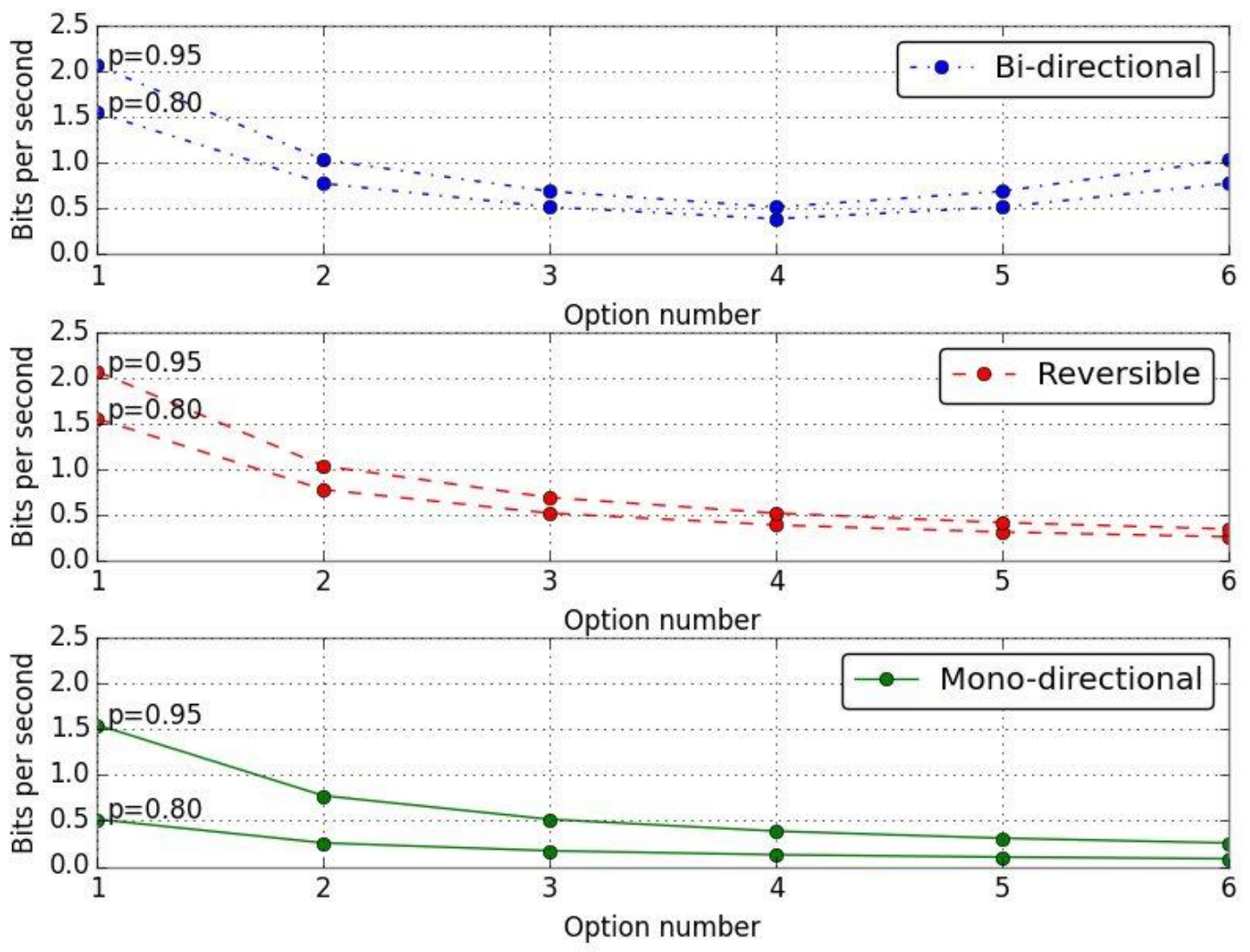

Figura 5. Comparação da utilidade (Utility) para $N=6$ opções.

A curva bidirecional sugere que o desempenho irá reduzir de uma forma mais suave para os indivíduos com menos precisão. Por outro lado, a navegação unidirecional é utilizável apenas com altos valores de precisão. Este poderia ser o caso de um controle biológico muito eficiente, mas na maioria das situações, a taxa de erro é significativa, cerca de $10-15 \%$. Além disso, a grande variabilidade no desempenho entre os indivíduos leva a observar baixa precisão em, pelo menos, alguns indivíduos. 


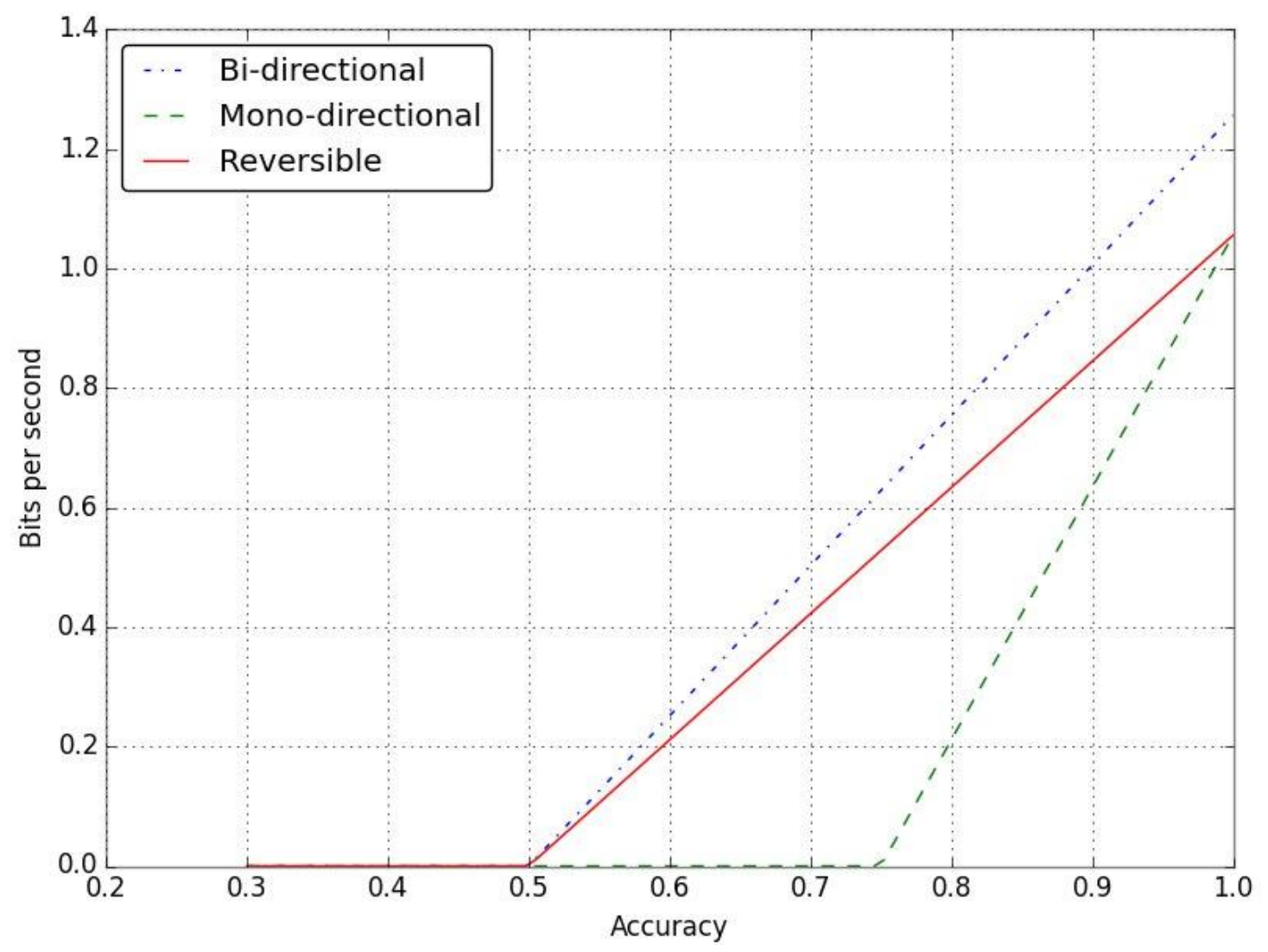

Figura 6. Comparação entre a Utility média utilizando as três abordagens de navegação para um menu com $\mathrm{N}=6$ opções.

\section{Conclusões}

Nesse trabalho foi desenvolvida uma interface de controle para um sistema assistivo baseado em sinais biológicos lidos de uma cadeira de rodas. O estudo foi conduzido baseado no princípio de melhorar a experiência do usuário com o sistema proposto e o desempenho geral.

BCI Utility foi o método escolhido para medir o desempenho do sistema,o qual se mostrou mais adequado do que a informação clássica de taxa de transferência de informação (ITR), uma vez que leva em consideração o comportamento da interface de controle.

O trabalho também demonstrou que umaInterface de Controle com três comandos fornece umbom equilíbrio entre a simplicidade, conveniência do usuário e eficácia. AIC foi desenvolvida de modo independente do tradutor de sinais biológicos, a fim de proporcionar uma interface flexível e extensível.

Além disso, através da construção de interface de um modo independente, permite-se a utilização do sistema por meio de diferentes sinais biológicos.

A IC fará parte de um sistema assistivo multimodal destinado a interagir com um ambiente inteligente, e será possível para o usuário escolher a técnica mais eficaz, dependendo do grau da sua incapacidade. 


\subsection{Trabalhos Futuros}

Na próxima etapa do trabalho será avaliada uma proposta baseada em um menu de referência para sistemas de controle operados por sinais biológicos. Tal valor de referência inclui a métrica Utility, bem como outros aspectos da experiência do usuário; exemplos desses aspectos é a fadiga do usuário e o seu engajamento. Assim, será fornecida uma maneira confiável e repetível para quantificar tais aspectos e comparar diferentes tecnologias ou técnicas de um ponto de vista centrado no usuário.

Em seguidaa interface será estendida para mais aplicações, em queserá aumentado o número de cômodos com possibilidade de interação pelo usuário e também o número de equipamentos disponíveis no sistema, além da implementação da navegação da cadeira de rodas pelo ambiente inteligente.

\section{Agradecimentos}

Os autores agradecem à Universidade Federal do Espírito Santo (UFES) pela estrutura de laboratórios e equipamentos oferecidos, ao Conselho Nacional de Desenvolvimento Científico e Tecnológico $(\mathrm{CNPq})$ e à Coordenação de Aperfeiçoamento de Pessoal de Nível Superior (CAPES) pelo apoio financeiro e pelas bolsas concedidas.

\section{Referências}

Bissoli, A. L. C., Ferrara, F., Silveira, M. L. e Bastos Filho, T. F. (2015a) Interação com o Ambiente e Comando de Equipamentos por Infravermelho para Cadeirantes. In $1^{\text {st }}$ International Workshop on Assistive Technology, p. 177-180.

Bissoli, A. L. C., Ferrara, F., Sime, M. M. e Bastos Filho, T. F. (2015b) A Multimodal Assistive System to Operate a Smart Environment. In $1^{\text {st }}$ International Workshop on Assistive Technology, p. 103-106.

Cook, A and Polgar, J. (2013) "Cook and Hussey's assistive technologies: principles and practice.” Elsevier Health Sciences.

Kirkup, L. et al. (1997) "EEG-based system for rapid on-off switching without prior learning." Medical and Biological Engineering and Computing 35.5, p. 504-509.

Mason, S.and Birch, G. (2003) "A general framework for brain-computer interface design." Neural Systems and Rehabilitation Engineering, IEEE Transactions on 11.1, p. $70-85$.

Seno, B., Matteucci, M. and Mainardo, L. (2010) "The utility metric: a novel method to assess the overall performance of discrete brain-computer interfaces." Neural Systems and Rehabilitation Engineering, IEEE Transactions on 18.1,p. 20-28.

Wolpaw, J. et al.(2002) "Brain-computer interfaces for communication and control."Clinical neurophysiology 113.6, p. 767-791. 